\title{
New COVID-19 Cases and Hospitalizations Among Adults, by Vaccination Status - New York, May 3-July 25, 2021
}

\begin{abstract}
Eli S. Rosenberg, $\mathrm{PhD}^{1,2}$; David R. Holtgrave, $\mathrm{PhD}^{2}$; Vajeera Dorabawila, $\mathrm{PhD}^{1}$; MaryBeth Conroy, $\mathrm{MPH}^{1}$; Danielle $\mathrm{Greene}$, DrPH ${ }^{1}$; Emily Lutterloh, MD ${ }^{1,2}$; Bryon Backenson, MS ${ }^{1,2}$; Dina Hoefer, $\mathrm{PhD}^{1}$; Johanne Morne, MS ${ }^{1}$; Ursula Bauer, PhD ${ }^{1}$; Howard A. Zucker, MD, JD ${ }^{1}$
\end{abstract}

\section{On August 18, 2021, this report was posted as an MMWR Early Release on the MMWR website (https://www.cdc.gov/mmwr).}

Data from randomized clinical trials and real-world observational studies show that all three COVID-19 vaccines currently authorized for emergency use by the Food and Drug Administration* are safe and highly effective for preventing COVID-19-related serious illness, hospitalization, and death $(1,2)$. Studies of vaccine effectiveness (VE) for preventing new infections and hospitalizations attributable to SARS-CoV-2, the virus that causes COVID-19), particularly as the B.1.617.2 (Delta) variant has become predominant, are limited in the United States (3). In this study, the New York State Department of Health linked statewide immunization, laboratory testing, and hospitalization databases for New York to estimate rates of new laboratory-confirmed COVID-19 cases and hospitalizations by vaccination status among adults, as well as corresponding VE for full vaccination in the population, across all three authorized vaccine products. During May 3-July 25, 2021, the overall age-adjusted VE against new COVID-19 cases for all adults declined from $91.8 \%$ to $75.0 \%$. During the same period, the overall age-adjusted VE against hospitalization was relatively stable, ranging from $89.5 \%$ to $95.1 \%$. Currently authorized vaccines have high effectiveness against COVID-19 hospitalization, but effectiveness against new cases appears to have declined in recent months, coinciding with the Delta variant's increase from $<2 \%$ to $>80 \%$ in the U.S. region that includes New York and relaxation of masking and physical distancing recommendations. To reduce new COVID-19 cases and hospitalizations, these findings support the implementation of a layered approach centered on vaccination, as well as other prevention strategies such as masking and physical distancing.

Four databases (the Citywide Immunization Registry, New York State Immunization Information System, Electronic Clinical Laboratory Reporting System, and Health Electronic Response Data System [HERDS]) were linked to construct a surveillance-based cohort of adults aged $\geq 18$ years residing in New York by using individual name-based identifiers, date of birth, and zip code of residence. The Citywide Immunization Registry and the New York State Immunization Information

\footnotetext{
*As of the publication date of this report, COVID-19 vaccines by PfizerBioNTech, Moderna, and Janssen (Johnson \& Johnson) have been authorized by the Food and Drug Administration under Emergency Use Authorization.
}

System are used to collect and store all COVID-19 provider vaccination data for persons residing in New York City and the rest of the state, respectively (excluding selected settings such as Veterans Affairs and military health care facilities); persons were considered fully vaccinated $\geq 14$ days after receipt of the final vaccine dose. ${ }^{\dagger}$ The Electronic Clinical Laboratory Reporting System collects all reportable COVID-19 test results (nucleic acid amplification test [NAAT] or antigen) in New York (4); a new COVID-19 case was defined as the receipt of a new positive SARS-CoV-2 NAAT or antigen test result, but not within 90 days of a previous positive result. HERDS includes a statewide, daily electronic survey of all inpatient facilities in New York; new admissions with a laboratory-confirmed COVID-19 diagnosis are entered into HERDS daily by trained hospital staff members.

After a period of phased COVID-19 vaccine eligibility based on age, occupation, setting, or comorbidities beginning in December 2020, all New York residents aged $\geq 60$ years were eligible for vaccination by March 10, 2021; eligibility was expanded to persons aged $\geq 30$ years by March 30 , and to all adults aged $\geq 18$ years by April $6 .{ }^{\S}$ To allow time for a large portion of vaccinated persons to achieve full immunity, this study was restricted to the week beginning May 3 through the week beginning July 19, 2021.

Breakthrough infections were defined as new cases among persons who were fully vaccinated on the day of specimen collection. Hospitalizations among persons with breakthrough infection were defined as new hospital admissions among persons fully vaccinated on the reporting day. The total adult state population that was fully vaccinated and unvaccinated was assessed for each day and stratified by age group (18-49 years, 50-64 years, and $\geq 65$ years). Persons who were partially vaccinated were excluded from analyses. For each week and age group, the rates of new cases and hospitalizations were calculated among fully vaccinated and unvaccinated persons, by respectively dividing the counts for each group by the

\footnotetext{
$\dagger$ Final dose was the second dose for Pfizer-BioNTech and Moderna vaccines, first dose for Janssen vaccine.

$\$$ https:/www.governor.ny.gov/news/governor-cuomo-announces-new-yorkers30-years-age-and-older-will-be-eligible-receive-covid-19

The total adult state population that was unvaccinated was calculated as the total U.S. Census population, minus fully or partially vaccinated persons. Persons who were partially vaccinated were defined as those who initiated a vaccine series but did not complete it or were within 14 days after completion.
} 
fully vaccinated and unvaccinated person-days in that week. Age-adjusted VE each week was estimated as the populationweighted mean of the age-stratified VE. ${ }^{* *}$ The interval between completing vaccination and positive SARS-CoV-2 test result date was summarized using the median, interquartile range (IQR), and percentage tested $\geq 7$ days from being fully vaccinated. ${ }^{\dagger \dagger}$ The ratio of hospitalizations to cases was computed for each vaccination group to understand the relative severity of cases. Statistical testing was not performed because the study included the whole population of interest and was not a sample.

By July 25, 2021, a total of 10,145,974 (65.6\%) New York adults aged $\geq 18$ years were fully vaccinated; $860,640(5.6 \%)$ were partially vaccinated. Among fully vaccinated adults, $51.3 \%$ had received Pfizer-BioNTech, 39.9\% had received Moderna, and $8.8 \%$ had received Janssen (Johnson $\&$ Johnson) vaccines. During May 3-July 25, a total of 9,664 new cases (1.31 per 100,000 person-days) occurred among fully vaccinated adults, compared with 42,507 (9.80 per 100,000 person-days) among unvaccinated adults (Table). Most (97.8\%) new cases among fully vaccinated persons occurred $\geq 7$ days after being classified fully vaccinated $($ median $=77$ days; $\mathrm{IQR}=49-103)$. During May 3-July 25, case rates among fully vaccinated persons were

\footnotetext{
** For both outcomes, VE at each week and age group was calculated as 1-(Ratevaccinated / Rateunvaccinated).

$\dagger \dagger$ The percentage tested $\geq 7$ days from being fully vaccinated was included to inform possible undiagnosed infection before full vaccination was achieved.
}

generally similar across age groups, as were case rates among unvaccinated persons, declining through the end of June before increasing in July (Figure 1). Weekly estimated VE against new laboratory-confirmed infection during May 3-July 25 for all age groups generally declined, going from $91.8 \%$ to $71.6 \%$ for persons aged $18-49$ years, $92.9 \%$ to $78.0 \%$ for persons aged $50-64$ years, and $90.5 \%$ to $80.0 \%$ for persons aged $\geq 65$ years. During May 3-July 25, the overall, age-adjusted VE against infection declined from $91.8 \%$ to $75.0 \%$ (Figure 1) (Table).

A total of 1,285 new COVID-19 hospitalizations (0.17 per 100,000 person-days) occurred among fully vaccinated adults, compared with 7,288 (1.68 per 100,000 person-days) among unvaccinated adults (Table). Hospitalization rates generally declined through the week of July 5 , but increased the weeks of July 12 and July 19, and were higher among fully vaccinated and unvaccinated persons aged $\geq 65$ years compared with younger age groups (Figure 2). Age group-specific estimated VE against hospitalization remained stable, ranging from $89.1 \%$ to $97.1 \%$ for persons aged $18-49$ years, from $89.8 \%$ to $96.1 \%$ for persons aged $50-64$ years, and from $86.5 \%$ to $94.2 \%$ for persons aged $\geq 65$ years. During May $3-$ July 25 , the overall, age-adjusted $\mathrm{VE}$ against hospitalization was generally stable from $89.5 \%$ to $95.1 \%$ (Figure 2) (Table). The ratio of hospitalizations to cases was moderately lower among fully vaccinated (13.3 hospitalizations per 100 cases) compared with unvaccinated (17.1 hospitalizations per 100 cases) groups.

TABLE. Vaccination coverage, new COVID-19 cases, and new hospitalizations with laboratory-confirmed COVID-19 among fully vaccinated and unvaccinated adults, and estimated vaccine effectiveness - New York, May 3-July 25, 2021

\begin{tabular}{|c|c|c|c|c|c|c|c|c|c|c|c|c|c|}
\hline \multirow[b]{3}{*}{ Week starting } & \multicolumn{3}{|c|}{ Population* } & \multicolumn{4}{|c|}{ New cases ${ }^{\dagger}$} & \multirow{3}{*}{$\begin{array}{c}\text { Estimated } \\
\text { vaccine } \\
\text { effectiveness, \% }\end{array}$} & \multicolumn{5}{|c|}{ New hospitalizations $§$} \\
\hline & \multirow{2}{*}{$\begin{array}{c}\text { Average no. } \\
\text { fully vaccinated }\end{array}$} & \multirow{2}{*}{$\begin{array}{l}\text { Average no. } \\
\text { unvaccinated }\end{array}$} & \multirow{2}{*}{$\begin{array}{c}\text { Full } \\
\text { vaccination } \\
\text { coverage, } \%\end{array}$} & \multicolumn{2}{|c|}{$\begin{array}{c}\text { Fully } \\
\text { vaccinated }\end{array}$} & \multicolumn{2}{|c|}{ Unvaccinated } & & \multicolumn{2}{|c|}{$\begin{array}{c}\text { Fully } \\
\text { vaccinated } \\
\end{array}$} & \multicolumn{2}{|c|}{ Unvaccinated } & \multirow{2}{*}{$\begin{array}{l}\text { Estimated vaccine } \\
\text { effectiveness, \% }\end{array}$} \\
\hline & & & & No. & Rate* $^{*}$ & No. & Rate $^{*}$ & & No. & Rate $^{*}$ & No. & Rate* & \\
\hline May 3 & $6,225,937$ & $6,176,926$ & 40.2 & 685 & 1.57 & 8,853 & 20.47 & 91.8 & 157 & 0.36 & 1,474 & 3.41 & 94.3 \\
\hline May 10 & $6,918,649$ & $5,929,937$ & 44.7 & 579 & 1.20 & 6,733 & 16.22 & 92.4 & 147 & 0.30 & 1,143 & 2.75 & 94.0 \\
\hline May 17 & $7,610,155$ & $5,655,798$ & 49.2 & 542 & 1.02 & 4,703 & 11.88 & 91.2 & 133 & 0.25 & 968 & 2.45 & 95.1 \\
\hline May 24 & $8,190,035$ & $5,409,414$ & 52.9 & 428 & 0.75 & 3,059 & 8.08 & 90.8 & 140 & 0.24 & 748 & 1.98 & 92.4 \\
\hline May 31 & $8,658,888$ & $5,247,446$ & 56.0 & 359 & 0.59 & 2,244 & 6.11 & 90.2 & 89 & 0.15 & 549 & 1.49 & 93.4 \\
\hline Jun 7 & $9,002,566$ & $5,106,617$ & 58.2 & 345 & 0.55 & 1,627 & 4.55 & 87.9 & 99 & 0.16 & 443 & 1.24 & 91.4 \\
\hline Jun 14 & $9,240,752$ & $4,983,758$ & 59.7 & 342 & 0.53 & 1,338 & 3.84 & 86.0 & 89 & 0.14 & 320 & 0.92 & 89.5 \\
\hline Jun 21 & $9,484,737$ & $4,875,026$ & 61.3 & 395 & 0.59 & 1,288 & 3.77 & 83.6 & 63 & 0.09 & 283 & 0.83 & 92.6 \\
\hline Jun 28 & $9,715,900$ & $4,779,103$ & 62.8 & 542 & 0.80 & 1,510 & 4.51 & 80.4 & 71 & 0.10 & 286 & 0.85 & 91.6 \\
\hline Jul 5 & $9,881,062$ & $4,696,156$ & 63.9 & 941 & 1.36 & 2,325 & 7.07 & 78.7 & 73 & 0.11 & 269 & 0.82 & 92.5 \\
\hline Jul 12 & $10,003,980$ & $4,608,129$ & 64.6 & 1,725 & 2.46 & 3,323 & 10.30 & 73.1 & 90 & 0.13 & 339 & 1.05 & 92.9 \\
\hline Jul 19 & $10,105,628$ & $4,509,581$ & 65.3 & 2,781 & 3.93 & 5,504 & 17.44 & 75.0 & 134 & 0.19 & 466 & 1.48 & 93.6 \\
\hline Total & - & - & - & 9,664 & 1.31 & 42,507 & 9.80 & - & 1,285 & 0.17 & 7,288 & 1.68 & - \\
\hline
\end{tabular}

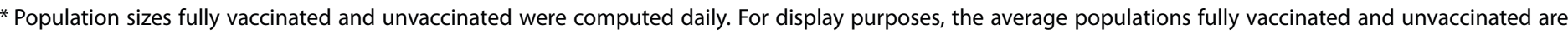

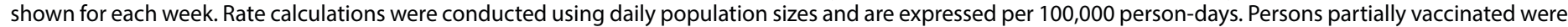
excluded from analyses.

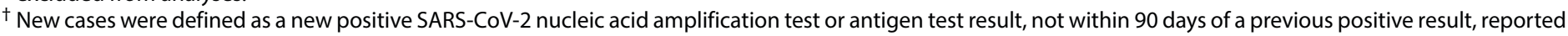
to the Electronic Clinical Laboratory Reporting System, which collects all reportable COVID-19 test results in New York.

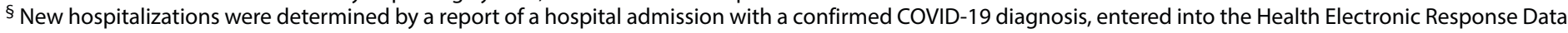
System, which includes a statewide, daily electronic survey of all inpatient facilities in New York.

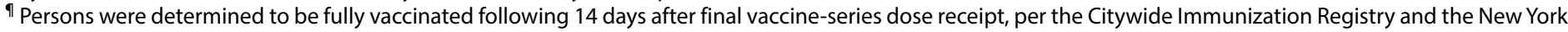

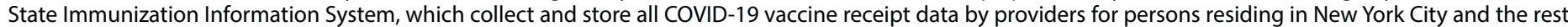
of New York, respectively. 


\section{Discussion}

In this study, current COVID-19 vaccines were highly effective against hospitalization (VE $>89 \%$ ) for fully vaccinated New York residents, even during a period during which prevalence of the Delta variant increased from $<2 \%$ to $>80 \%$ in the U.S. region that includes New York, societal public health restrictions eased, $\$ ₫$ and adult full-vaccine coverage in

$\$ \$$ https://covid.cdc.gov/covid-data-tracker/\#variant-proportions

FIGURE 1. New COVID-19 cases among fully vaccinated and unvaccinated adults, vaccine coverage, and estimated vaccine effectiveness, by age New York, May 3-July 25, 2021
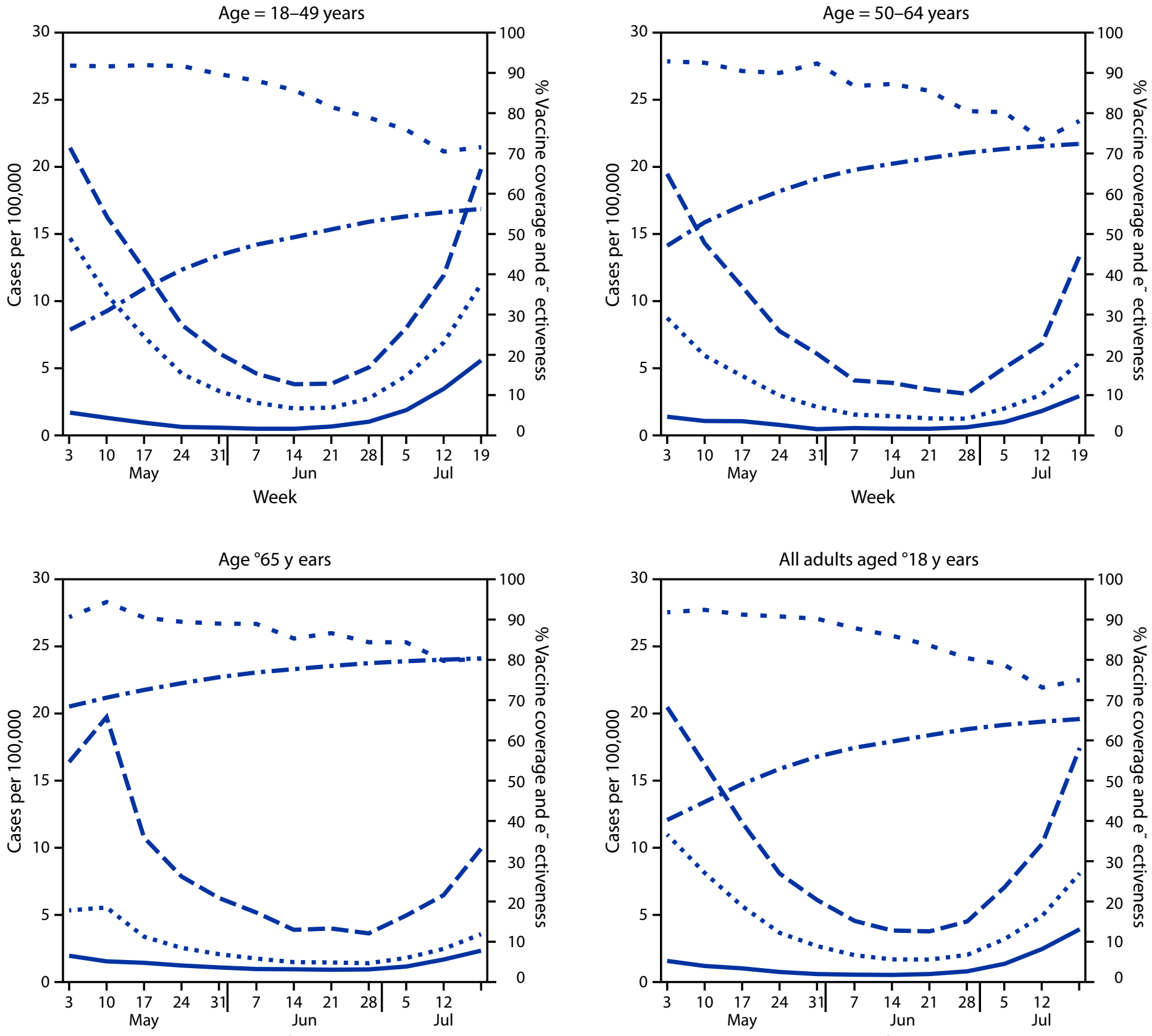

Week

Week

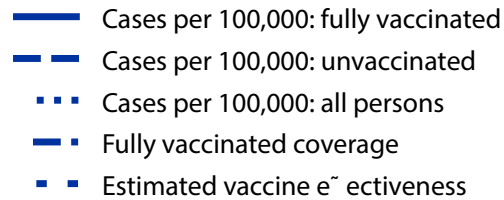


New York reached 65\%. However, during the assessed period, rates of new cases increased among both unvaccinated and fully vaccinated adults, with lower relative rates among fully vaccinated persons. Moreover, VE against new infection declined from $91.8 \%$ to $75.0 \%$. To reduce new COVID-19 cases and hospitalizations, these findings support the implementation of a layered approach centered on vaccination, as well as other prevention strategies.

FIGURE 2. New hospitalizations with laboratory-confirmed COVID-19 among fully vaccinated and unvaccinated adults, vaccine coverage, and estimated vaccine effectiveness, by age - New York, May 3-July 25, 2021
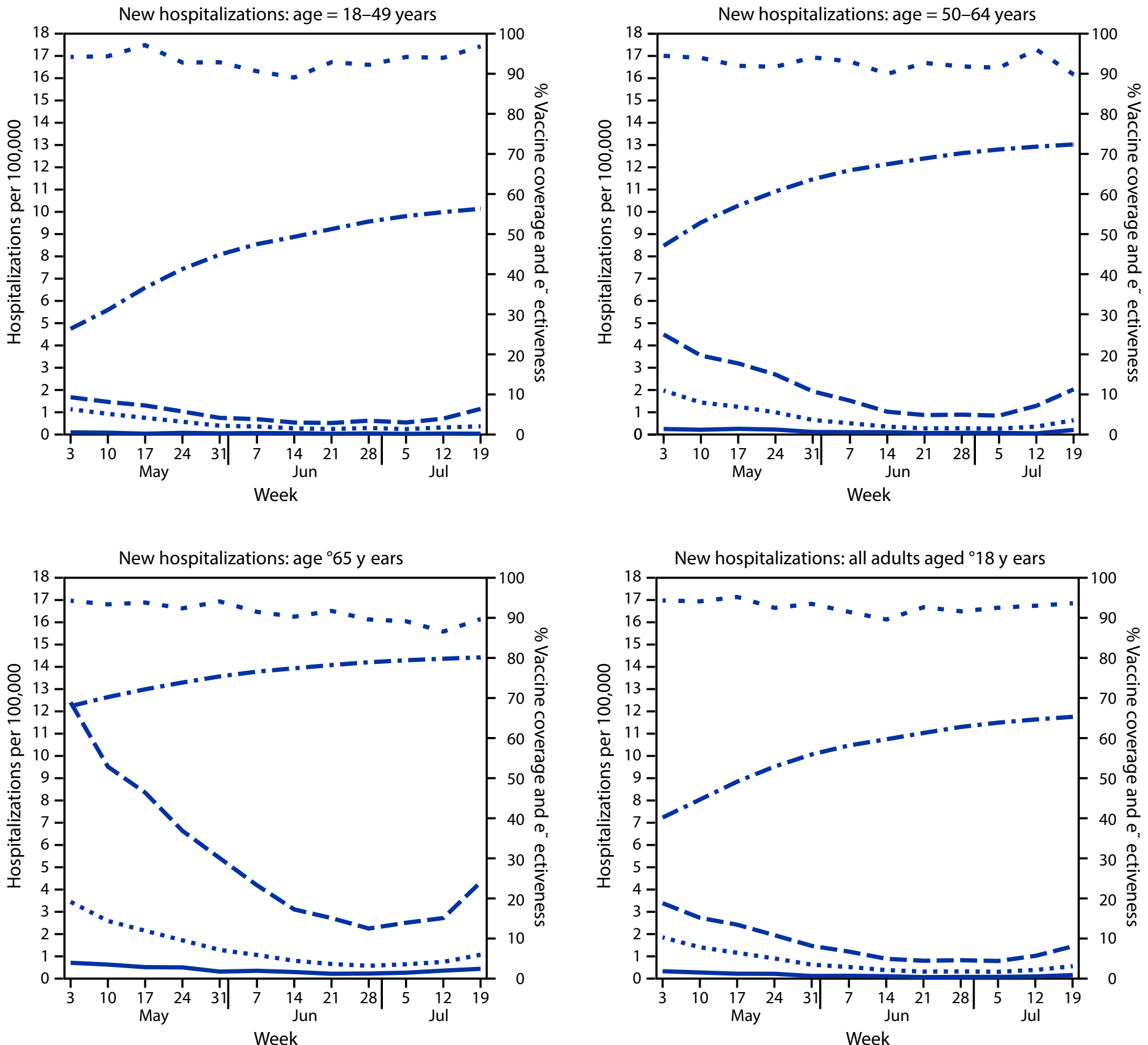

\footnotetext{
Hospitalizations per 100,000: fully vaccinated

- Hospitalizations per 100,000: unvaccinated

[- Hospitalizations per 100,000: all persons

- Fully vaccinated coverage

- Estimated vaccine $e^{\sim}$ ectiveness
} 


\section{Summary}

What is already known about this topic?

Real-world studies of population-level vaccine effectiveness against laboratory-confirmed SARS-CoV-2 infection and COVID-19 hospitalizations are limited in the United States.

What is added by this report?

During May 3-July 25, 2021, the overall age-adjusted vaccine effectiveness against hospitalization in New York was relatively stable $89.5 \%-95.1 \%)$. The overall age-adjusted vaccine effectiveness against infection for all New York adults declined from $91.8 \%$ to $75.0 \%$.

What are the implications for public health practice?

These findings support the implementation of multicomponent approach to controlling the pandemic, centered on vaccination, as well as other prevention strategies such as masking and physical distancing.

The findings from this study are consistent with those observed in other countries. Israel has reported $90 \%$ VE for the Pfizer-BioNTech vaccine against hospitalization; however, a decline in $\mathrm{VE}$ against new diagnosed infections occurred during June 20-July 17 (decreasing to $<65 \%$ ) (5). Another study in the United Kingdom found higher VE against infection with the Delta variant for Pfizer-BioNTech $(88 \%)$, which was lower than VE against the B.1.1.7 (Alpha) variant (94\%) (6).

The factors driving the apparent changes in VE, including variations by age, are uncertain. Changes in immune protection from current vaccine product dosing regimens are under investigation, 99 with additional doses being considered (7). Increased Delta variant viral load might underpin its increased transmissibility and could potentially lead to reduced vaccineinduced protection from infection (8). Further, variations from clinical trial findings could be because the trials were conducted during a period before the emergence of new variants and when nonpharmaceutical intervention strategies (e.g., wearing masks and physically distancing) were more stringently implemented, potentially lessening the amount of virus to which persons were exposed. Other factors that could influence VE include indirect protective effects of unvaccinated persons by vaccinated persons and an increasing proportion of unvaccinated persons acquiring some level of immunity through infection (9).

The findings in this report are subject to at least six limitations. First, although limiting the analysis period to after universal adult vaccine eligibility and age stratification likely helped to reduce biases, residual differences between fully vaccinated and unvaccinated groups have the potential to reduce estimated VE. Second, the analysis excluded partially vaccinated persons, to robustly assess $\mathrm{VE}$ for fully vaccinated

\footnotetext{
99 https://www.medrxiv.org/content/10.1101/2021.07.28.21261159v1
}

compared with that of unvaccinated persons. A supplementary sensitivity analysis that included partially vaccinated persons as unvaccinated yielded conservative $\mathrm{VE}$ for laboratory-confirmed infection (declining from $89.0 \%$ to $71.4 \%$ ) and for hospitalizations (ranging from $87.7 \%$ to $93.6 \%$ ). Third, exact algorithms were used to link databases; some persons were possibly not linked because matching variables were entered differently in the respective systems. Fourth, this study did not estimate VE by vaccine product, and persons were categorized fully vaccinated at 14 days after final dose, per CDC definitions; however, the Janssen vaccine might have higher efficacy at 28 days. ${ }^{* * *}$ Given that Janssen vaccine recipients accounted for $9 \%$ of fully vaccinated persons and the observed time period from full vaccination to infection (median 77 days), this would minimally affect the findings. Fifth, information on reasons for testing and hospitalization, including symptoms, was limited. However, a supplementary analysis found that among 1,285 fully vaccinated adults and 7,288 unvaccinated adults, 553 (43.0\%) and 4,231 (58.1\%), respectively, were reported to have been admitted for COVID-19 by hospital staff members using nonstandardized definitions. A sensitivity analysis of hospitalization VE limited to those admitted for COVID-19, found similar results (VE range $=92.5 \%-96.8 \%$ ), suggesting that the extent of bias was limited. Finally, data were too sparse to reliably estimate VE for COVID-19-related deaths.

This study's findings suggest currently available vaccines have high effectiveness for preventing laboratory-confirmed SARS-CoV-2 infection and COVID-19 hospitalization. However, VE against infection appears to have declined in recent months in New York, coinciding with a period of easing societal public health restrictions ${ }^{\dagger \dagger}$ and increasing Delta variant circulation (8). These findings support a multipronged approach to reducing new COVID-19 hospitalizations and cases, centered on vaccination, and including other approaches such as masking and physical distancing.

\section{*** https://www.fda.gov/media/146338/download \\ ${ }^{\dagger \dagger \dagger}$ https://coronavirus.jhu.edu/data/state-timeline/new-confirmed-cases/ new-york/205}

\section{Acknowledgments}

Steven Davis, Rebecca Hoen, New York State Department of Health; Citywide Immunization Registry Program, New York City Department of Health and Mental Hygiene.

Corresponding author: Eli Rosenberg, eli.rosenberg@health.ny.gov.

\footnotetext{
${ }^{1}$ New York State Department of Health; ${ }^{2}$ University at Albany School of Public Health, State University of New York, Rensselaer, New York.
}

All authors have completed and submitted the International Committee of Medical Journal Editors form for disclosure of potential conflicts of interest. No potential conflicts of interest were disclosed. 


\section{References}

1. Food and Drug Administration. COVID-19 vaccines. Silver Spring, MD: US Department of Health and Human Services; Food and Drug Administration; 2021. Accessed July 29, 2021. https://www.fda.gov/ emergency-preparedness-and-response/coronavirus-disease-2019covid-19/covid-19-vaccines.

2. Pilishvili T, Fleming-Dutra KE, Farrar JL, et al.; Vaccine Effectiveness Among Healthcare Personnel Study Team. Interim estimates of vaccine effectiveness of Pfizer-BioNTech and Moderna COVID-19 vaccines among health care personnel-33 U.S. sites, January-March 2021. MMWR Morb Mortal Wkly Rep 2021;70:753-8. PMID:34014909 https://doi.org/10.15585/mmwr.mm7020e2

3. CDC. COVID-19 vaccine breakthrough case investigation and reporting. Atlanta, GA: US Department of Health and Human Services, CDC; 2021. Accessed July 29, 2021. https://www.cdc.gov/vaccines/covid-19/ health-departments/breakthrough-cases.html

4. Rosenberg ES, Dufort EM, Blog DS, et al.; New York State Coronavirus 2019 Response Team. COVID-19 testing, epidemic features, hospital outcomes, and household prevalence, New York State-March 2020. Clin Infect Dis 2020;71:1953-9. PMID:32382743 https://doi. org/10.1093/cid/ciaa549
5. Israel Ministry of Health. Decline in vaccine effectiveness against infection and symptomatic illness. Jerusalem, Israel: Israel Ministry of Health; 2021. Accessed July 29, 2021. https://www.gov.il/en/departments/ news/05072021-03.

6. Lopez Bernal J, Andrews N, Gower C, et al. Effectiveness of Covid-19 vaccines against the B.1.617.2 (Delta) variant. N Engl J Med 2021. Epub July 21, 2021. PMID:34289274 https://doi.org/10.1056/ NEJMoa2108891

7. Kamar N, Abravanel F, Marion O, Couat C, Izopet J, Del Bello A. Three doses of an mRNA Covid-19 vaccine in solid-organ transplant recipients. N Engl J Med 2021. Epub June 23, 2021. PMID:34161700 https://doi. org/10.1056/NEJMc2108861

8. Brown CM, Vostok J, Johnson H, et al. Outbreak of SARS-CoV-2 infections, including COVID-19 vaccine breakthrough infections, associated with large public gatherings-Barnstable County, Massachusetts, July 2021. MMWR Morb Mortal Wkly Rep 20211;70:1059-62. PMID:34351882 https://doi.org/10.15585/mmwr.mm7031e2

9. Patel MK, Bergeri I, Bresee JS, et al. Evaluation of post-introduction COVID-19 vaccine effectiveness: summary of interim guidance of the World Health Organization. Vaccine 2021;39:4013-24. PMID:34119350 https://doi.org/10.1016/j.vaccine.2021.05.099 